\title{
Restoration of left ventricular geometry and improvement of left ventricular function in a rodent model of chronic ischemic cardiomyopathy
}

\author{
Jiashing Yu, BS, ${ }^{a}$ Karen L. Christman, PhD, ${ }^{a}$ Eric Chin, BS, ${ }^{c}$ Richard E. Sievers, BS, ${ }^{c}$ Maythem Saeed, PhD, ${ }^{\mathrm{b}}$ and \\ Randall J. Lee, MD, $\mathrm{PhD}^{\mathrm{a}, \mathrm{c}}$
}

\begin{abstract}
Objectives: Various approaches to myocardial reconstruction have been developed for the treatment of congestive heart failure resulting from ischemic cardiomyopathy.
\end{abstract}

\begin{abstract}
Methods: In this study we determined whether in situ application of polymers could reshape left ventricular geometry in a chronic rodent model of ischemic cardiomyopathy.

Results: We demonstrate that alginate and fibrin can augment left ventricular wall thickness, resulting in reconstruction of left ventricular geometry and improvement of cardiac function. Echocardiographic results at 5 weeks after injection of alginate demonstrated persistent improvement of left ventricular fractional shortening and prevention of a continued enlargement of left ventricular dimensions, whereas fibrin glue demonstrated no progression of left ventricular negative remodeling. There was increased arteriogenesis in both the alginate and fibrin glue groups compared with that seen in the phosphate-buffered saline control group. Infarct size was significantly reduced in the fibrin group $(P<.05)$, and there was a trend toward a smaller myocardial infarction in the alginate group.
\end{abstract}

Conclusion: Intramyocardially injected polymers can be used to reshape the aneurysmal left ventricle and might therefore be an approach for myocardial reconstruction and a potential option in treating chronic heart failure in human subjects.

Heart failure is a major health problem in the world. Currently, therapeutic approaches in treating patients with chronic heart failure include pharmacologic therapies, mechanical devices, and surgical intervention, ${ }^{1,2}$ whereas heart transplantation remains the only viable solution for endstage congestive heart failure. ${ }^{3}$

Cardiac tissue engineering aims to repair damaged myocardium by combing cell biology, material science, and engineering principles. ${ }^{4,5}$ Myocardial infarction (MI) results in left ventricular (LV) dilation, wall thinning, fibrosis, and reduced cardiac performance. One major concept of tissue engineering is to take into account the effect of the extracellular matrix (ECM). ECM scaffolds not only provide mechanical support for tissue but also regulate cell function. ${ }^{6}$ Engineered cardiac constructs composed of scaffold materials alone or in combination with cells or growth factors have

From the University of California Berkeley and University of California San Francisco

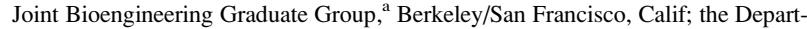
ment of Radiology, ${ }^{\mathrm{b}}$ University of California, San Francisco, Calif; and the Cardiovascular Research Institute and Department of Medicine, ${ }^{\mathrm{c}}$ University of California, San Francisco, Calif

Research was partially supported by grants from the Nora Eccles Treadwell Foundation and Symphony Medical, Inc.

Received for publication April 21, 2008; revisions received July 23, 2008; accepted for publication Aug 21, 2008.

Address for reprints: Randall J. Lee, MD, PhD, 500 Parnassus Ave, Cardiac Electrophysiology, MU East Tower, Box 1354. San Francisco, CA 94143 (E-mail: lee@ medicine.ucsf.edu).

J Thorac Cardiovasc Surg 2009;137:180-7

$0022-5223 / \$ 36.00$

Copyright (c) 2009 by The American Association for Thoracic Surgery

doi:10.1016/j.jtcvs.2008.08.036 been widely investigated. ${ }^{4} \mathrm{~A}$ range of materials from synthetic materials, such as polyglycolic acid, polylactic acid, or polyethylene glycol, to biologic materials, such as gelatin, collagen, or Matrigel (BD Biosciences, Franklin Lakes, NJ), have been used for myocardial repair. ${ }^{4} \mathrm{Re}-$ cently, polymers have been shown to be effective as an in situ tissue-engineering approach for preserving cardiac function after an acute MI. ${ }^{4}$ Compared with a bioengineered cardiac graft, the injectable scaffolds remain in liquid form until after injection. After solidification in vivo, the engineered biopolymer matrices have the potential to provide mechanical support and promote cell migration and proliferation and angiogenesis. ${ }^{6-9}$

The use of tissue-engineering techniques for myocardial repair has focused on tissue repair after an acute MI. In this study we investigated and compared the therapeutic effects of fibrin and alginate in a rodent model of chronic ischemic cardiomyopathy. We demonstrated that fibrin and alginate alone could reconstruct a LV aneurysm, improve cardiac function, reduce infarct scar percentage, and stimulate neovascularization. All these findings underline the potential of injectable biopolymers in cardiac tissue engineering for myocardial repair.

\section{MATERIALS AND METHODS Biopolymers}

Fibrin glue (CROSSEAL Fibrin Sealant; Omrix Biopharmaceuticals, Inc, New York, NY) consists of 2 components: biologic active components and thrombin. Biologic active components consist mainly of human fibrinogen, and thrombin is a highly specific protease that transforms the 


\author{
Abbreviations and Acronyms \\ $\mathrm{ECM}=$ extracellular matrix \\ $\mathrm{LV}=$ left ventricular \\ LVID = left ventricular internal dimension \\ MI = myocardial infarction \\ MRI = magnetic resonance imaging \\ PBS = phosphate-buffered saline \\ RGD $=$ arginine-glycine-asparagine
}

fibrinogen into fibrin. Alginate solution (1.5\%) was made from dissolving a high mannuronic acid (M units) alginate (ProNova LVM; FMC BioPolymer, Haugesund, Norway) in $0.9 \% \mathrm{NaCl}$. Alginate gel formation was based on the addition of the cross-linker solution, $102 \mathrm{mmol} / \mathrm{L} \mathrm{CaCl}_{2}{ }^{10}$

\section{Rat Chronic MI Model}

The animal protocol was approved by the Committee for Animal Research of the University of California San Francisco and was performed in accordance with the recommendations of the American Association for Accreditation of Laboratory Animal Care. This ischemia-reperfusion model has been described extensively in our previous studies. ${ }^{9,11}$ Female SpragueDawley rats (225-250 g) underwent 20 minutes of left anterior descending artery occlusion, followed by reperfusion. The chest was then closed, and the animal was allowed to recover for 5 weeks to allow for the development of a LV aneurysm. ${ }^{9}$

\section{Injection Operations}

Sprague-Dawley rats underwent left coronary artery occlusion for 20 minutes followed by reperfusion to test the hypothesis that a fibrin or alginate scaffold thickens the thinned wall of the aneurysmal left ventricle and reshapes LV geometry. Five weeks after infarction, at which time the remodeling process is largely complete, injections of either the control $(0.5 \%$ bovine serum albumin in phosphate-buffered saline [PBS], $\mathrm{n}=$ $10)$, fibrin $(n=10)$, or alginate $(n=10)$ were made directly into the infarcted myocardium. Biopolymers were delivered as 2 components with a Duploject applicator (Baxter, Deerfield, Ill), which holds the 2 components fibrin and thrombin for the fibrin group and sodium alginate and calcium chloride for the alginate group in separate syringes and provides simultaneous mixing and delivery. All injections were made through 27gauge needles into the infarcted area of the left ventricle. The infarct area was identified by a darker region of the LV wall with reduced contractility, mostly within the anterior wall. Three animals from each group $(n=3)$ were killed 24 hours after injection to examine the location and structural effect of the polymer injections compared with control results. Animals were perfused with $3 \mathrm{~mol} / \mathrm{L} \mathrm{KCl}$ before death to arrest the hearts in the diastolic phase of contraction.

To test the effects of in situ application of biopolymers in a chronic aneurysmal MI, the remainder of the animals (PBS, $n=7$; fibrin, $n=7$; and alginate, $\mathrm{n}=7$ ) were allowed to recover and survived for an additional 5 weeks after the injection (10-week-old MI) instead of dying at 24 hours after injection.

\section{Echocardiography}

Transthoracic echocardiographic analysis was performed on all animals after achievement of anesthesia with isoflurane $(2 \mathrm{~L} / \mathrm{min}) 5$ weeks after MI as a baseline echocardiogram. Follow-up echocardiograms were obtained 2 days and 5 weeks after injection (10 weeks after MI). The methodology of echocardiography used has been previously described and has demonstrated reproducibility and accuracy in a rat MI model. ${ }^{9,12}$ Fractional shortening (FS) as a measure of systolic function was calculated as follows:

$$
F S(\%)=[(L V I D d-L V I D s) / L V I D d] \times 100 \%,
$$

where $L V I D$ represents the LV internal dimension, $d$ represents diastole, and $s$ represents systole. An echocardiographer blinded to the treatment group acquired the images and performed the data analysis.

\section{Histology}

After the echocardiogram at 10 weeks after MI, the rats were killed with a pentobarbital overdose $(200 \mathrm{mg} / \mathrm{kg})$. The hearts were rapidly excised and fresh frozen in O.C.T. freezing medium (Sakura Finetek, Torrance, Calif) and were then sectioned into $10-\mu \mathrm{m}$ slices. Ten representative slides were stained with hematoxylin and eosin and Masson's trichrome stain (Accustain; Sigma, St Louis, Mo). Morphologic assessment of infarct size and LV wall thickness was quantified based on planimetry on Masson's trichrome-stained slides with SPOT 4.0.5 software (Diagnostic Instruments, Sterling Heights, Mich), as previously described. ${ }^{13}$ The infarcted area and left ventricle were traced. The infarct size was determined by the scar area divided by the LV area and was reported as a percentage of the total left ventricle. The segmental distribution of wall thickness was determined by measuring the anterior, lateral, posterior, and septal walls of each individual slide, taking the average of 10 slides for each heart.

\section{Immunohistochemistry and Immunofluorescence}

Five slides equally distributed through the infarct area were selected to examine the extent of the inflammatory response. The slides were fixed in acetone for 15 minutes and incubated in avidin, biotin, Sniper, and Eraser blocking solutions (Biocare Medical, Concord, Calif). The slides were stained with mouse anti-CD68 monocyte/macrophage monoclonal antibody (dilution 1:50; Chemicon International, Temecula, Calif) by using immunohistochemistry and diaminobenzidine stain (Animal Research Kit; DAKO, Glostrup, Denmark). The degree of inflammatory response was semiquantified by using a scoring method with scores of 0 to 4 ( 0 , nothing; 1 , few; 2 , moderate; 3 , much; 4 , most). Another 5 slides were taken to examine angiogenesis in the infarct area. The slides were fixed in $1.5 \%$ formaldehyde and then blocked with staining buffer $(0.3 \%$ Triton X-100 and $2 \%$ normal goat serum in PBS). Sections were stained with anti- $\alpha$ smooth muscle actin monoclonal antibody (dilution 1:400, Sigma) and goat anti-mouse IgG2a (dilution 1:1000; Molecular Probes, Eugene, Ore). Positive smooth muscle staining with a visible lumen of a diameter 10 to $100 \mu \mathrm{m}$ within the infarct was used as the criterion to calculate arteriole density. ${ }^{13}$

\section{Statistical Analysis}

Data were reported as the mean \pm standard deviation. A paired $t$ test was used for comparing baseline and postinjection echocardiographic data for animals serving as internal controls. Echocardiographic data across groups, histologic assessment, and immunostaining were compared by using 1-way analysis of variance with Holm's adjustment.

\section{RESULTS}

\section{Acute Injection of Biopolymers Reshapes the Aneurysmal LV Wall}

In those animals killed 24 hours after injection, fibrin glue and alginate were observed within the infarct wall. At the location of injection, both fibrin glue and alginate markedly altered the LV geometry, resulting in an LV cavity geometry more resembling the control geometry. The reshaping of the LV wall was not seen with the PBS control (Figure 1). 

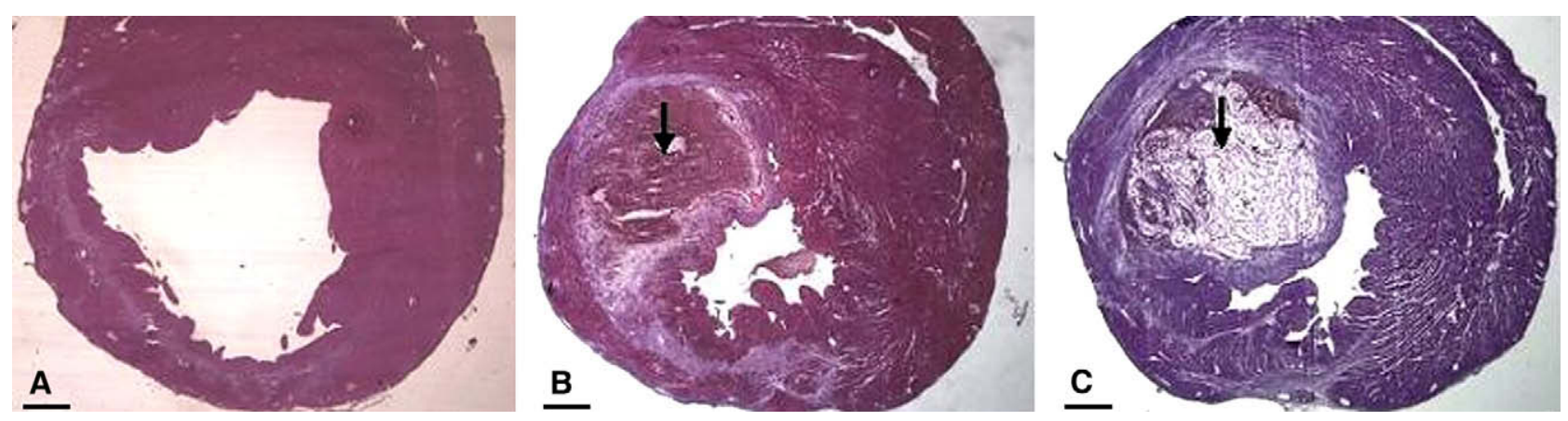

FIGURE 1. Hematoxylin and eosin stain shows the presence of biopolymers in situ after 24 hours of injection. A, Infarcted myocardium treated with PBS. B, Infarcted myocardium treated with fibrin glue. C, Infarcted myocardium treated with alginate. The black arrow indicates the location of biopolymers. Scale bars $=1.0 \mathrm{~mm}$.

\section{In Situ Application of Biopolymers Improves LV Function}

The functional effects of fibrin glue and alginate were investigated by means of echocardiographic analysis at baseline, 2 days after treatment, and 5 weeks after treatment. The summary of echocardiographic data is provided in Figure 2.

The baseline ( 5 weeks after MI) data indicated that there were no difference between groups in LV function, LV internal dimensions (LVIDs), and LV wall thickness. However, 2 days after the injection of the biopolymers, improved cardiac function and increased wall thickness were seen, and these values were significantly increased compared with baseline values $(P<.05)$. The fractional shortening of the alginate group increased from $27.5 \pm 9.3$ to $41.7 \pm 11.7(P=$ $.04)$, and that in the fibrin group increased from $29.3 \pm$ 6.0 to $40.8 \pm 5.2(P<.01)$. The anterior systolic wall thickness of the alginate group increased from $1.74 \pm 0.67$ to 3.60 $\pm 1.25(P<.01)$, and that of the fibrin group increased from $1.90 \pm 0.54$ to $3.24 \pm 0.74(P<.01)$. The anterior diastolic wall thickness also increased significantly after biopolymer injection (alginate group, $P<.01$; fibrin group, $P=.02$ ). LVID during systole decreased from $0.51 \pm 0.15$ to 0.36 $\pm 0.17(P<.01)$ in the alginate group and from $0.51 \pm$ 0.08 to $0.38 \pm 0.05(P<.02)$ in the fibrin group. LVID during diastole also decreased in both groups, from $0.73 \pm 0.10$ to $0.60 \pm 0.15(P=.01)$ and $0.72 \pm 0.05$ to $0.65 \pm 0.06$ $(P=.01)$ for the alginate and fibrin groups, respectively.

Echocardiographic analysis performed at 5 weeks after injection (10 weeks after MI) demonstrated a significant continuation of $\mathrm{LV}$ dilatation in the control group and a worsening LV function compared with that before injection. The fractional shorting of the control PBS group deteriorated from $25.6 \pm 10.6$ to $22.4 \pm 8.5, \mathrm{LV}$ dimension enlarged $(0.59 \pm$ 0.11 to $0.66 \pm 0.14 \mathrm{~cm}, P$ systole $=.003 ; 0.79 \pm 0.06$ to $0.85 \pm 0.10 \mathrm{~cm}, P$ diastole $=.02)$, and wall thickness decreased significantly $(1.69 \pm 0.67$ to $1.45 \pm 0.52 \mathrm{~cm}, P$ systole $=.09 ; 1.15 \pm 0.35$ to $0.97 \pm 0.26 \mathrm{~cm}, P$ diastole $=.02)$. Similarly, the fibrin-treated group demonstrated deterioration of LV fractional shortening. The fractional shortening of the fibrin group deteriorated to $23.1 \pm 7.5$, as did LV systolic dimension $(0.51 \pm 0.08$ to $0.58 \pm 0.11 \mathrm{~cm}, P$ systole $=.02)$. However, LV diastolic dimension and wall thickness did not significantly change. In contrast, the trend with alginate was toward improved LV function (fractional shortening from $27.5 \% \pm 9.3 \%$ to $33.5 \% \pm 14.1 \%$ ), significantly increased wall thickness $(1.74 \pm 0.67$ to $2.63 \pm 1.03 \mathrm{~cm}, P$ systole $<$ $.01 ; 1.34 \pm 0.63$ to $1.90 \pm 0.86 \mathrm{~cm}, P$ diastole $=.02$ ), and preserved LV dimensions.

\section{Histology}

Distinctive red muscle fibers and blue collagen deposition were seen 10 weeks after MI in all treatment groups (Figure 3, $A-C)$. Tissue composed of mainly collagen covered the entire LV myocardium in all animals, demonstrating the consistency and reproducibility of the MI model in this laboratory. Figure 3, D, showed the comparison of infarct percentage 5 weeks after the treatments. The control PBS group exhibited a $16 \% \pm 3 \%$ infarction. The fibrin group had significantly smaller infarct size $(12 \% \pm 2 \%, P=.04)$ compared with the control group. The alginate group also had a trend, although not significant, toward reduction of infarct size $(14 \% \pm 5 \%)$ compared with the control group. The LV anterior, lateral, posterior, and septal wall thicknesses were measured (Figure 3,E). The LV walls of the alginate and fibrin groups were significantly thicker than those of the control group in the anterior, lateral, and posterior segments $(P<$ $.05)$. The anterior wall of the alginate group was thicker than that of the fibrin group, whereas there were no statistical differences in the lateral and posterior segments. All groups had similar wall thicknesses in septal segments.

In addition, there was detection of alginate after 5 weeks of injection, whereas fibrin was completely reabsorbed (Figure $3, B$ and $C$ ), indicating the slower degradation rate and persistence of alginate compared with fibrin.

\section{Neovascularization}

Smooth muscle $\alpha$-actin staining was used as an indicator for arterioles. Individual arterioles within the infarct area 

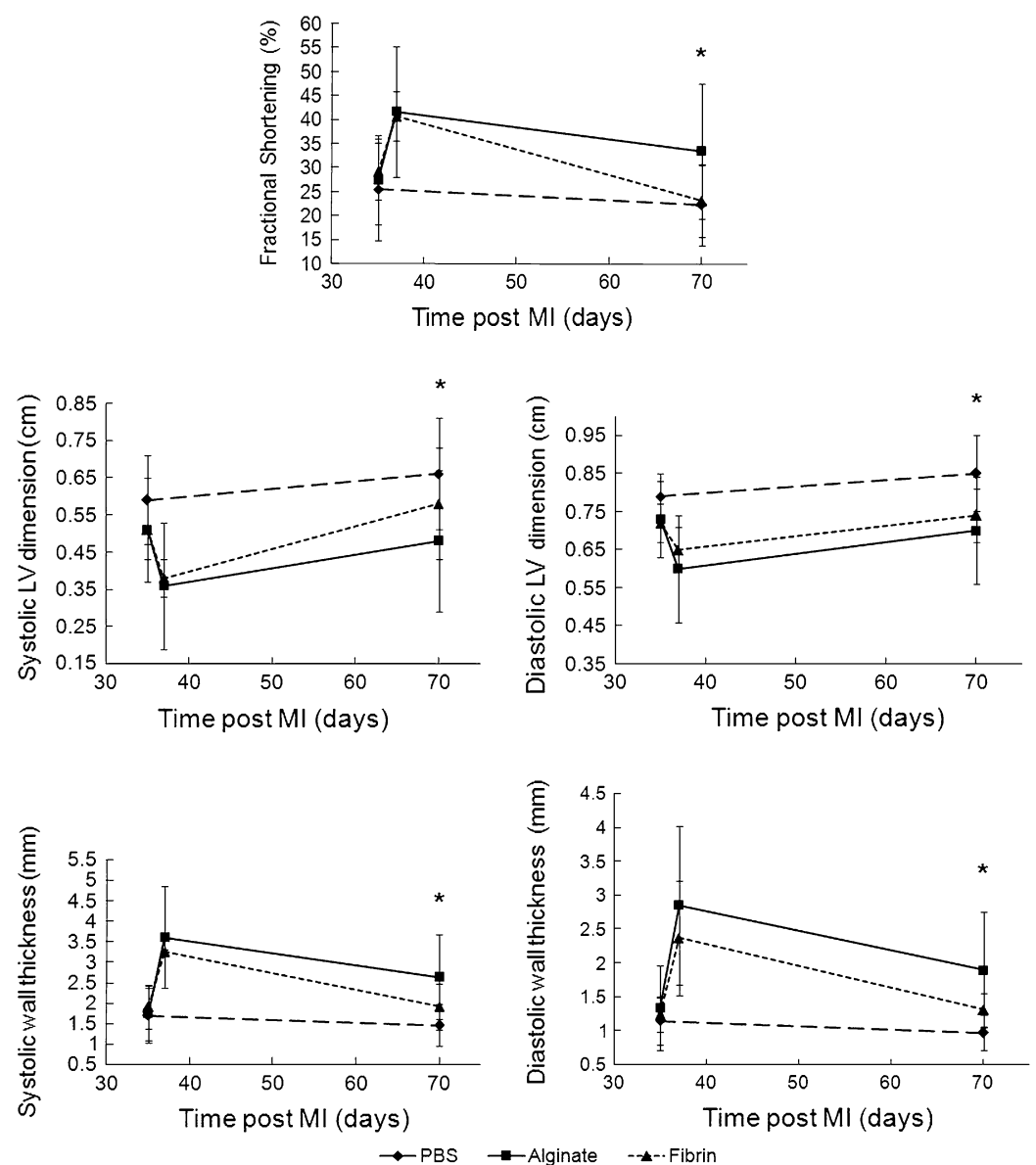

FIGURE 2. Echocardiographic data. *Significant difference between groups $(P<.05)$ at 5 weeks after injection $(10$ weeks after myocardial infarction $[M I])$. $L V$, Left ventricular; $P B S$, phosphate-buffered saline.

were counted, and the total arteriole number divided by infarct area was reported as arteriole density. Figure 4, A through $C$, showed the arteriogenesis in the middle of the infarct region and border zone in contrast to normal myocardium. Both fibrin $\left(24 \pm 7 / \mathrm{mm}^{2}\right)$ and alginate $(22 \pm 8 /$ $\mathrm{mm}^{2}$ ) showed significantly higher arteriole density than PBS $\left(14 \pm 1 / \mathrm{mm}^{2}\right)$, indicating that biopolymers have the ability to stimulate neovascularization in situ. Figure $5 \mathrm{dem}-$ onstrates that new arteriole formation occurs in the collagen deposition zone of the infarcted myocardium.

\section{Inflammation}

Anti-CD68 antibody was used to detect the presence of macrophages after 5 weeks of injection to evaluate whether the biopolymer would cause a sustained inflammatory response. There were $\mathrm{CD} 68^{+}$cells clustering in all specimens, indicating that inflammation might chronically exist in the infarct area, regardless of the treatment. The fibrin was entirely reabsorbed at 5 weeks after injection, and thus there was no more traceable biopolymer scaffold to locate the implanted scaffold and inflammatory cells. There were few scattered macrophages in the vicinity of alginate. The overall average inflammatory cells in all treatment groups were about the same, with a score lower than 1 (approximately $10 \%$ of the area), indicating that there was no significant difference between groups.

\section{DISCUSSION}

These experiments demonstrate that intramyocardial injections of polymers can be used to shape the aneurysmal $\mathrm{LV}$, improve LV function, and promote preservation of LV wall thickness. Biopolymer scaffolds mechanically provide structural support for chronic LV aneurysms and can potentially be used as an acellular approach for myocardial reconstruction. Previous cardiac tissue-engineering studies focused mainly on in vitro cardiac grafts. ${ }^{14-16}$ Cells were seeded in the polymer scaffold and subsequently patched onto the surface of the infarcted myocardium. However, engineered cardiac patches require invasive operations and are constrained by limitation of nutrients and oxygen supply. Unlike in vitro engineered cardiac tissue, injectable biopolymers can be engineered to allow the host itself to act as a bioreactor and reconstruct the damaged tissue in situ. 

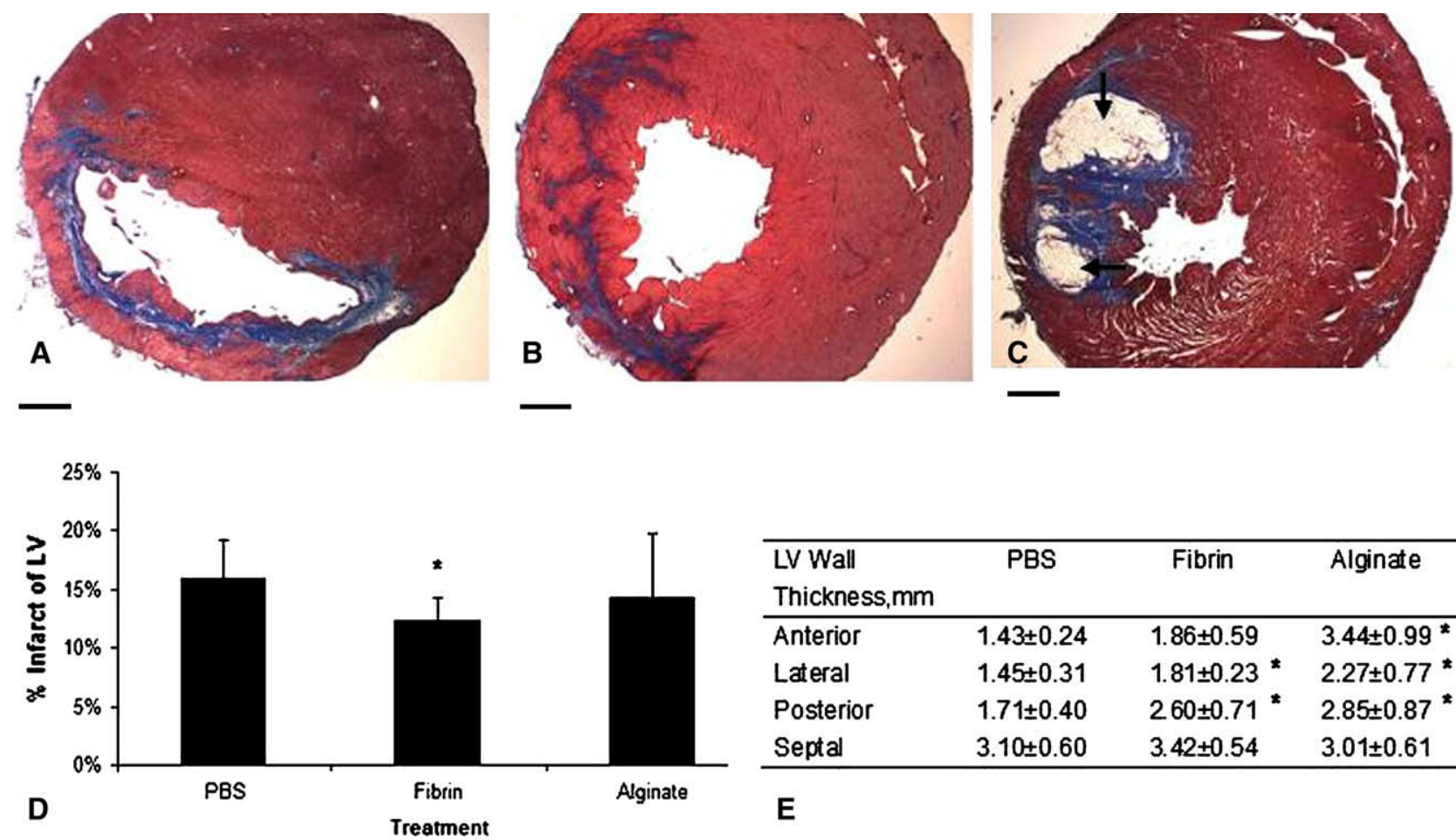

\begin{tabular}{lccl}
\hline $\begin{array}{l}\text { LV Wall } \\
\text { Thickness,mm }\end{array}$ & PBS & Fibrin & Alginate \\
\hline Anterior & $1.43 \pm 0.24$ & $1.86 \pm 0.59$ & $3.44 \pm 0.99^{*}$ \\
Lateral & $1.45 \pm 0.31$ & $1.81 \pm 0.23^{*}$ & $2.27 \pm 0.77^{*}$ \\
Posterior & $1.71 \pm 0.40$ & $260 \pm 0.71 *$ & $2.85 \pm 0.87^{*}$ \\
Septal & $3.10 \pm 0.60$ & $3.42 \pm 0.54$ & $3.01 \pm 0.61$ \\
\hline
\end{tabular}

E

FIGURE 3. Histologic assessment 5 weeks after injection. Masson's trichrome staining showed the collagen deposition (blue) in the infarcted areas of the phosphate-buffered saline (PBS; A)-, fibrin (B)-, and alginate (C)-injected groups. Black arrows indicate the existence of alginate. The infarcted percentage of the left ventricle $(L V ; \mathrm{D})$ and wall thickness $(\mathrm{E})$ were measured. Scale bars $=1.0 \mathrm{~mm}$.

In addition to physically integrating into the defective myocardium, scaffold materials must be able to withstand the load of the tissue and provide mechanical strength. ${ }^{17,18}$

Jackson and colleagues ${ }^{19}$ applied a modified Laplace law calculation of wall stress on an ovine anteroapical MI model. The results indicated that geometric changes cause increased dynamic wall stress, which likely contributes to border-zone expansion and remodeling. The introduction of biopolymers into the thin LV wall alters the geometry of the aneurysmal myocardium and potentially decreases myocardial stress, resulting in decreased dilatation of the left ventricle. Interestingly, the present results corroborate results from computer modeling studies that indicate implanting noncontractile materials into damaged myocardium might introduce beneficial effects. Wall and associates ${ }^{20}$ used a novel finiteelement analysis to predict the effect of injection of a material into the myocardium. Their results indicate that the injection of a small volume of noncontractile material into infarcted myocardium can decrease end-systolic fiber stress. Moreover, in the infarct injection improves ejection fraction and the stroke volume/end-diastolic volume relationship but has no effect on the Starling relationship.

The histologic and echocardiographic results show that both fibrin and alginate are able to restore the geometry of a chronic aneurysmal left ventricle and increase LV function 2 days after injection of the biopolymer. The long-term increase in LV fractional shortening appeared to be at least partially related to the persistence of the polymer, presumably providing sustained physical support. At 5 weeks after the injection of fibrin glue, LV fractional shortening was similar to control LV fractional shortening. Histologic analysis revealed the persistence of alginate, whereas fibrin glue had been reabsorbed (Figure 3). Evidence supporting the notion that fibrin has a beneficial effect, even after it is reabsorbed, is the preservation of LV wall thickness and diastolic LV dimensions.

The ischemia-reperfusion rat model of MI is a frequently used model for assessing ischemic preconditioning, cardioprotective drugs, and MI remodeling. ${ }^{21-23}$ The ischemiareperfusion MI produces a more mottled infarct compared with a transmural total ligation MI and is clinically representative of an MI seen with a reperfused coronary vessel. ${ }^{24}$ The aneurysms produced by ischemia-reperfusion are located either at the apex or on the anterior surface, as commonly seen in patients with an anterior MI. ${ }^{25}$ The ischemia-reperfusion rat model has been used to investigate the efficacy of cells and polymers for myocardial reconstruction and can be used as an initial model to test tissue-engineering strategies for myocardial repair and regeneration. ${ }^{11}$ However, the altering effects of biopolymers on the material properties of the ventricular wall are more easily tested in large-animal MI models. 

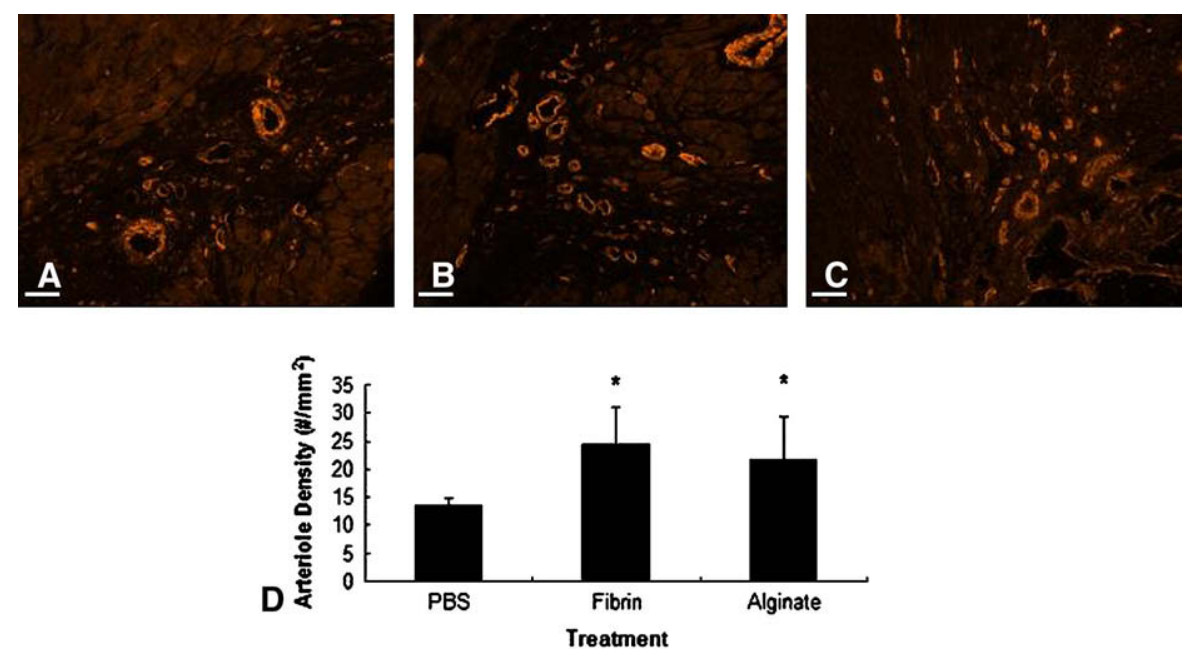

FIGURE 4. Increased arteriole density in the infarcted area. Smooth muscle actin staining showed visible arterioles of the phosphate-buffered saline $(P B S)(A)$, fibrin (B), and alginate (C) groups. Quantitative analysis of arteriole density is presented in panel D. Scale bars $=100 \mu \mathrm{m}$.

Recently, magnetic resonance imaging (MRI) tagging techniques were exploited on tracking LV wall motion and stress/strain distribution of the LV wall. Walker and coworkers ${ }^{26}$ evaluated end-diastolic stress on a sheep model after linear repair of LV aneurysm with MRI-based finiteelement stress analysis. A potential future study is to examine the effect of biomaterials after MI with this MRI technique.

Ideally, biopolymer scaffolds should both physically reside in and interact with the host tissue. Biomaterials not only coexist with the tissue but can also influence the cardiac microenvironment at a molecular level, which is critical for tissue regeneration. ${ }^{27}$ Previous studies in our laboratory demonstrated that fibrin glue enhanced angiogenesis in an acute MI model. ${ }^{13}$ In this chronic MI study, we found that fibrin glue stimulated new blood vessel formation, as seen in previous acute studies. Interestingly, alginate also promoted angiogenesis, although not as dramatically as fibrin glue. This result implies that even after the pathologic remodeling process, the introduction of matrix materials can still influence cell activities, such as cell recruitment, migration, and proliferation.

The mechanisms of the angiogenic effect of fibrin might be due to its molecular properties. ${ }^{28}$ Fibrin has binding domains for various growth factors, such as fibroblast growth factor, which is an angiogenic factor. Moreover, the fibrin fragment $E$ has been shown to stimulate neovascularization in vitro. ${ }^{29}$ Fibrin glue also contains an arginine-glycineasparagine (RGD) sequence; RGD is a ubiquitous cell adhesion ligand that not only binds to integrins on cell surfaces but can also activate downstream signaling pathways. RGD activates $\alpha_{5} \beta_{3}$ integrin, which is involved in the activation of vascular endothelial growth factor receptor ${ }^{30}$; fibrinogen without RGD sequences does not to induce angiogenesis. ${ }^{31}$

Alginate is composed of simple linear polysaccharide copolymers and has been widely applied in the food and pharmaceutical industries. ${ }^{32}$ The lack of interaction between alginate and mammalian cells might be the major limitation
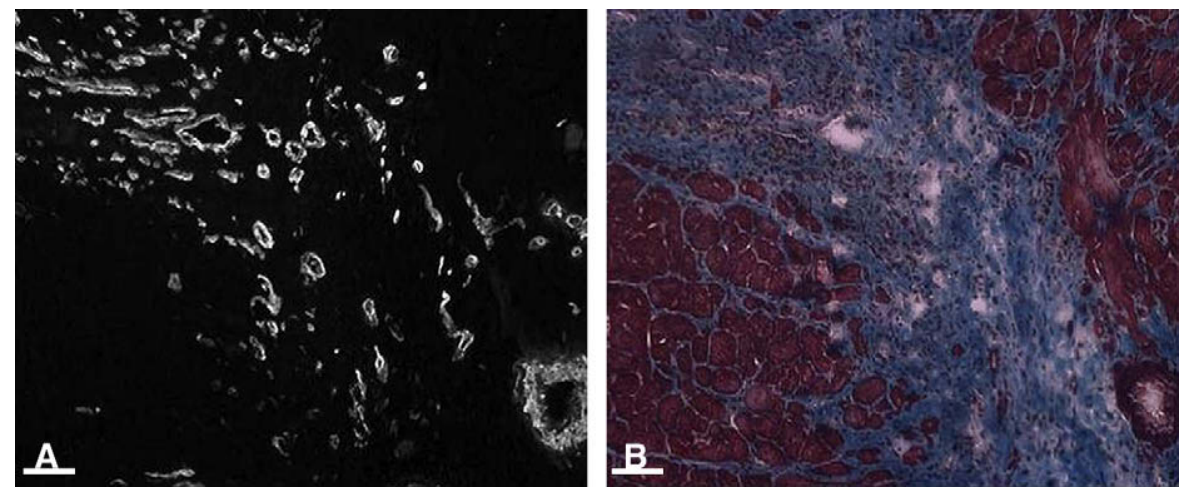

FIGURE 5. Arterioles in the collagen deposition area. Smooth muscle actin staining of arterioles in the infarcted zone (A) and corresponding Trichrome staining (B). Scale bars $=100 \mu \mathrm{m}$. 
for its use in tissue engineering. Possible mechanisms of the angiogenic effect of alginate include an inflammatory response of the biopolymers or a mechanical matrix effect of the biopolymer, which allows the migration of cells into the infarcted area. The result of CD68 staining showed that there was no difference in inflammatory assessment at 5 weeks after injection. There were inflammatory cells residing in the infarct area in all groups. The inflammatory cells might be recruited to the infarct area, mainly because of the injection surgery independent of treatments. Another possible mechanism could be the effect of ischemia-induced chemokines that regulate leukocytes recruitment during pathophysiologic remodeling in early MI. ${ }^{31,33}$

Future studies of in situ cardiac tissue-engineering approaches need to focus on optimization of the degradation rate and control of the material properties of scaffold materials. The durability of materials should be similar to the rate of tissue regeneration. ${ }^{17}$ Although fibrin undergoes reabsorption, the endogenous cells can invade into the fibrin scaffolds, secrete their own ECM, and perform cellular activities. ${ }^{34}$ The degradation rate of fibrin glue can be adjusted by changing the concentration of aprotinin, the proteinase that prevents proteolytic degradation and increases the mechanical strength of fibrin. ${ }^{35,36}$ Alginate is usually degraded by dissolution; the compressive and shear modulus depends on the ionic concentration and ratio of $\mathrm{G}$ to $\mathrm{M}$ units. ${ }^{37,38}$ Moreover, alginate can be modified by means of an oxidization technique, which accelerates the degradation rate of alginate without significantly changing its mechanical properties. ${ }^{39}$

This study demonstrates an acellular method for treating chronic MI with injectable fibrin or alginate. This comparative study could provide guidance for selecting suitable materials for delivering growth factors and genes or transplanting cells. The results also indicate that a noncellular construct itself might be sufficient for cardiac function improvement and angiogenesis.

\section{References}

1. Braunwald E, Bristow MR. Congestive heart failure: fifty years of progress. Circulation. 2000;102(suppl 4):IV14-23

2. Hunt SA. ACC/AHA 2005 guideline update for the diagnosis and management of chronic heart failure in the adult: a report of the American College of Cardiology/ American Heart Association Task Force on Practice Guidelines. J Am Coll Cardiol. 2006;47:1503-5.

3. el Oakley RM, Yonan NA, Simpson BM, Deiraniya AK. Extended criteria for cardiac allograft donors: a consensus study. J Heart Lung Transplant. 1996;15: 255-95.

4. Christman KL, Lee RJ. Biomaterials for the treatment of myocardial infarction. J Am Coll Cardiol. 2006;48:907-13.

5. Zammaretti P, Jaconi M. Cardiac tissue engineering: regeneration of the wounded heart. Curr Opin Biotechnol. 2004;15:430-4.

6. Huang NF, Lee RJ, Li S. Chemical and physical regulation of stem cells and progenitor cells: potential for cardiovascular tissue engineering. Tissue Eng. 2007;13: 1809-23.

7. Dai W, Wold LE, Dow JS, Kloner RA. Thickening of the infarcted wall by collagen injection improves left ventricular function in rats: a novel approach to preserve cardiac function after myocardial infarction. J Am Coll Cardiol. 2005;46: 714-9.
8. Kofidis T, de Bruin JL, Hoyt G, Lebl DR, Tanaka M, Yamane T, et al. Injectable bioartificial myocardial tissue for large-scale intramural cell transfer and functional recovery of injured heart muscle. J Thorac Cardiovasc Surg. 2004;128: 571-8.

9. Christman KL, Fok HH, Sievers RE, Fang Q, Lee RJ. Fibrin glue alone and skeletal myoblasts in a fibrin scaffold preserve cardiac function after myocardial infarction. Tissue Eng. 2004;10:403-9.

10. Awad HA, Wickham MQ, Leddy HA, Gimble JM, Guilak F. Chondrogenic differentiation of adipose-derived adult stem cells in agarose, alginate, and gelatin scaffolds. Biomaterials. 2004;25:3211-22.

11. Huang NF, Sievers RE, Park JS, Fang Q, Li S, Lee RJ. A rodent model of myocardial infarction for testing the efficacy of cells and polymers for myocardial reconstruction. Nat Protoc. 2006;1:1596-609.

12. Litwin SE, Katz SE, Morgan JP, Douglas PS. Serial echocardiographic assessment of left ventricular geometry and function after large myocardial infarction in the rat. Circulation. 1994;89:345-54.

13. Christman KL, Vardanian AJ, Fang Q, Sievers RE, Fok HH, Lee RJ. Injectable fibrin scaffold improves cell transplant survival, reduces infarct expansion, and induces neovasculature formation in ischemic myocardium. $J$ Am Coll Cardiol. 2004;44:654-60.

14. Li RK, Yau TM, Weisel RD, Mickle DA, Sakai T, Choi A, et al. Construction of a bioengineered cardiac graft. J Thorac Cardiovasc Surg. 2000;119:368-75.

15. Kofidis T, Akhyari P, Boublik J, Theodorou P, Martin U, Ruhparwar A, et al. In vitro engineering of heart muscle: artificial myocardial tissue. $J$ Thorac Cardiovasc Surg. 2002;124:63-9.

16. Kellar RS, Landeen LK, Shepherd BR, Naughton GK, Ratcliffe A, Williams SK. Scaffold-based three-dimensional human fibroblast culture provides a structural matrix that supports angiogenesis in infarcted heart tissue. Circulation. 2001; 104:2063-8.

17. Drury JL, Mooney DJ. Hydrogels for tissue engineering: scaffold design variables and applications. Biomaterials. 2003;24:4337-51.

18. Butler DL, Goldstein SA, Guilak F. Functional tissue engineering: the role of biomechanics. J Biomech Eng. 2000;122:570-5.

19. Jackson BM, Gorman JH 3rd, Salgo IS, Moainie SL, Plappert T, St JohnSutton M, et al. Border zone geometry increases wall stress after myocardial infarction: contrast echocardiographic assessment. Am J Physiol Heart Circ Physiol. 2003;284:H475-9

20. Wall ST, Walker JC, Healy KE, Ratcliffe MB, Guccione JM. Theoretical impact of the injection of material into the myocardium: a finite element model simulation. Circulation. 2006;114:2627-35.

21. Zhu BQ, Sievers RE, Browne AE, Lee RJ, Chatterjee K, Grossman W, et al. Comparative effects of aspirin with ACE inhibitor or angiotensin receptor blocker on myocardial infarction and vascular function. $J$ Renin Angiotensin Aldosterone Syst. 2003;4:31-7.

22. Murohara T, Guo JP, Lefer AM. Cardioprotection by a novel recombinant serine protease inhibitor in myocardial ischemia and reperfusion injury. $J$ Pharmacol Exp Ther. 1995;274:1246-53.

23. Fu Y, Wang Z, Chen WL, Moore PK, Zhu YZ. Cardioprotective effects of nitric oxide-aspirin in myocardial ischemia-reperfused rats. Am J Physiol Heart Circ Physiol. 2007;293:H1545-52.

24. Ferdinandy P, Schulz R, Baxter GF. Interaction of cardiovascular risk factors with myocardial ischemia/reperfusion injury, preconditioning, and postconditioning. Pharmacol Rev. 2007;59:418-5.

25. Gorlin R, Klein MD, Sullivan JM. Prospective correlative study of ventricular aneurysm. Mechanistic concept and clinical recognition. Am J Med. 1967;42: 512-31.

26. Walker JC, Ratcliffe MB, Zhang P, Wallace AW, Hsu EW, Saloner DA, et al. Magnetic resonance imaging-based finite element stress analysis after linear repair of left ventricular aneurysm. J Thorac Cardiovasc Surg. 2008;135: 1094-102.

27. Davis ME, Hsieh PC, Grodzinsky AJ, Lee RT. Custom design of the cardiac microenvironment with biomaterials. Circ Res. 2005;97:8-15.

28. Hall H, Baechi T, Hubbell JA. Molecular properties of fibrin-based matrices for promotion of angiogenesis in vitro. Microvasc Res. 2001;62:315-26.

29. Bootle-Wilbraham CA, Tazzyman S, Thompson WD, Stirk CM, Lewis CE. Fibrin fragment $\mathrm{E}$ stimulates the proliferation, migration and differentiation of human microvascular endothelial cells in vitro. Angiogenesis. 2001;4: 269-75.

30. Soldi R, Mitola S, Strasly M, Defilippi P, Tarone G, Bussolino F. Role of alphavbeta3 integrin in the activation of vascular endothelial growth factor receptor-2. EMBO J. 1999; 18:882-92. 
31. Vandervelde S, van Luyn MJ, Tio RA, Harmsen MC. Signaling factors in stem cell-mediated repair of infarcted myocardium. J Mol Cell Cardiol. 2005;39: 363-76.

32. Augst AD, Kong HJ, Mooney DJ. Alginate hydrogels as biomaterials. Macromol Biosci. 2006;6:623-33.

33. Frangogiannis NG. Chemokines in the ischemic myocardium: from inflammation to fibrosis. Inflamm Res. 2004;53:585-95.

34. Hotary KB, Yana I, Sabeh F, Li XY, Holmbeck K, Birkedal-Hansen H, et al. Matrix metalloproteinases (MMPs) regulate fibrin-invasive activity via MT1MMP-dependent and -independent processes. J Exp Med. 2002;195:295-308.

35. Yao L, Swartz DD, Gugino SF, Russell JA, Andreadis ST. Fibrin-based tissueengineered blood vessels: differential effects of biomaterial and culture param- eters on mechanical strength and vascular reactivity. Tissue Eng. 2005;11: 991-1003.

36. Chekanov VS, Nikolaychik VV, Rieder MA, Tchekanov GV, Hernandez I, Eisenstein R, et al. Autologous biological glue and aprotinin prevent ischemia in latissimus dorsi muscle after mobilization. Basic Appl Myol. 1998;8:211-9.

37. LeRoux MA, Guilak F, Setton LA. Compressive and shear properties of alginate gel: effects of sodium ions and alginate concentration. J Biomed Mater Res. 1999; 47:46-53.

38. Smidsrod O, Skjak-Braek G. Alginate as immobilization matrix for cells. Trends Biotechnol. 1990;8:71-8.

39. Alsberg E, Kong HJ, Hirano Y, Smith MK, Albeiruti A, Mooney DJ. Regulating bone formation via controlled scaffold degradation. J Dent Res. 2003;82:903-8. 